\title{
Explicit Approximation Solutions and Proof of Convergence of the Space-Time Fractional Advection Dispersion Equations
}

\author{
E. A. Abdel-Rehim \\ Department of Mathematics and Computer Science, Faculty of Science, Suez Canal University, Ismailia, Egypt \\ Email: entsarabdalla@hotmail.com
}

Received June 29, 2013; revised July 29, 2013; accepted August 6, 2013

Copyright (C) 2013 E. A. Abdel-Rehim. This is an open access article distributed under the Creative Commons Attribution License, which permits unrestricted use, distribution, and reproduction in any medium, provided the original work is properly cited.

\begin{abstract}
The space-time fractional advection dispersion equations are linear partial pseudo-differential equations with spatial fractional derivatives in time and in space and are used to model transport at the earth surface. The time fractional order is denoted by $\beta \in(0,1]$ and $\alpha \in(0,2]$ is devoted to the space fractional order. The time fractional advection dispersion equations describe particle motion with memory in time. Space-fractional advection dispersion equations arise when velocity variations are heavy-tailed and describe particle motion that accounts for variation in the flow field over entire system. In this paper, I focus on finding the precise explicit discrete approximate solutions to these models for some values of $0<\beta \leq 1$ with $0<\alpha<1,1<\alpha<2$ while the Cauchy case as $\alpha=1$ and the classical case as $\alpha=2$ with $\beta=1$ are studied separately. I compare the numerical results of these models for different values of $\alpha$ and $\beta$ and for some other related changes. The approximate solutions of these models are also discussed as a random walk with or without a memory depending on the value of $\beta$. Then I prove that the discrete solution in the Fourierlaplace space of theses models converges in distribution to the Fourier-Laplace transform of the corresponding fractional differential equations for all the fractional values of $\alpha$ and $\beta$.
\end{abstract}

Keywords: Advection-Dispersion Processes; Grünwald-Letnikov Scheme; Explicit Difference Schemes; Caputo Time-Fractional Derivative; Inverse Riesz Potential; Random Walk with and without a Memory; Convergence in Distributions; Fourier-Laplace Domain

\section{Introduction}

The development which has happened on the last twentyfive years on the fractional calculus opened many new applications on many fields such as physics, hydrodynamics, chemistry, financial mathematics, and some other fields. Actually a growing number of articles and books which are interesting on this field and its applications have appeared in these last 25 years (see for example: [1-5] and see also my thesis [6]. Fractional in time means that the first-order time derivative is replaced by the Caputo derivative of order $\beta \in(0,1]$, see [4]. Fractional in space means replacing the second order space-derivative is replaced by the Feller operator [7] in the symmetric case with order $0<\alpha \leq 2$.

The behaviour of particles in transport under the earth surface is an important problem. For examples, the transport of solute and contaminant particles in surface and subsurface water flows, the behaviour of soil particles and associated soil particles, and the transport of sediment particles and sediment-borne substances in turbulent flow. There are many other examples in this field. The classical advection dispersion equation, ade, has been used to formulate such problems. The generalized fractional advection-dispersion equation, fade, has recently gotten an increasing interest from many scientists because it has many applications specially on studying the transport of passive tracers carried by fluid flow in a porous medium, see Benson, Meerschaert et al. [8-12]. In their work they gave applications and experimental results for the space-fade.

There is no unique solution for the space-time fractional diffusion processes but there are some attempts using different forms of the hyper geometric functions, as for example: in [13] the authors attempted to find an 
analytical solution for other special form of the fractional, see also $[14,15]$. Therefore authors who study modelling of fractional processes use some developed methods to descritize the fractional operators. For examples, in [16], the authors used their own method of descretization to find the approximate solution of the space-fade and gave some numerical results. In [17], the authors studied the approximate solution of the space-fade, for $1<\alpha<2$ and $\beta=1$, only using the backward Grünwald-Letnikov Scheme. The backward Grünwald-Letnikov Scheme has been successfully adopting by Gorenflo, Mainrdi, and etal, see $[4,18,19]$ for modelling space-fractional diffusion processes. Also has been used by Gorenflo and E. A. Abdel-Rehim, see [20-24] for modelling time-fractional Fokker-Planck equations and their convergence in the Fourier Laplace domain.

I am interested in this paper to find the approximate solutions of the space-time fractional advection equation, space-time fade, by adopting the backward GrünwaldLetnikov Scheme joined with the common finite difference methods. The space-time fade is considered as a diffusion process under the action of a constant force in a fractal medium with a memory. I study and numerically investigate the effect of the time fractional on the path of the particle motion as well as the effect of the spacefractional order for the three cases as: $0<\alpha<1,1<\alpha<$ 2 , and $\alpha=1$. I compare between all these cases numerically. My numerical results are consistent with the results of [17] for the studied case $1<\alpha<2$ and $\beta=1$. The approximate solutions according to the values $1<\alpha$ $<2$, and $\alpha=1$ joined with $0<\beta \leq 1$ are firstly studied on this paper. The proof of the convergence in distribution for each case is also considered. Therefore this paper is organized as follows: Section 1 is denoted to the introduction; Section 2 is devoted to the definitions of the used fractional operators and their Laplace-Fourier transformations; and Section 3 introduces the classical case $\alpha=2, \beta=1$. Section 4 , the fractional in time $\alpha=2$, $0<\beta<1$, is studied. Section 5.1 is denoted to the case $0<\alpha<1,0<\beta \leq 1$, and $1<\alpha<2,0<\beta \leq 1$ is studied at Section 5.2. Section 6 is devoted to the $\operatorname{case} \alpha=1$, $0<\beta \leq 1$. Finally, the numerical results will be displayed and explained in Section 7 and one compares these results with the results of the given references.

\section{Important Definitions and the Outline of the Proof of Convergence in Distribution}

The generalized fade reads

$$
\left\{\begin{array}{l}
\underset{t *}{D^{\beta}} u(x, t)=a \underset{x 0}{D^{\alpha}} u(x, t)-b \frac{\partial}{\partial x} u(x, t) \\
0<\beta \leq 1,0<\alpha \leq 2, \alpha \neq 1 \\
u(x, 0)=\delta(x) \quad-R \leq x \leq R \\
u(-R, t)=u(R, t)=0 \quad 0 \leq t \leq T
\end{array}\right.
$$

Here $a$, and $b$ are positive constants representing the dispersion coefficient, and the average fluid velocity and it acts as the drift term to the right respectively. My aim is to give the approximation solutions of the space-time fad equations for all values of $\alpha$ and $\beta$. I study also the convergence of the approximation solutions to the solutions of the corresponding analytical solutions of the space-time $\mathrm{fad}$ equations in the Fourier-Laplace domain. The used time-fractional derivative operator $\underset{t *}{D^{\beta}} u(x, t)$ is called Caputo fractional operator, see [4] to know the relation between Caputo fractional derivative and the famous Riemann-Liouville fractional derivative operators. Caputo fractional derivative in the Laplace domain reads

$$
\mathcal{L}\left\{D_{*}^{\beta} f(t) ; s\right\}=s^{\beta} \tilde{f}(s)-s^{\beta-1} f(0) s>0 .
$$

This equation is important for solving the fractional differential equations because it show the dependence on the initial conditions. Here $\frac{\partial^{\alpha} u(x, t)}{\partial x^{\alpha}}=D_{x 0}^{\alpha} u(x, t)$ is called the Riesz space-fractional differentiation operator. I adopt here the notation introduced by [25]. It is formally a power of the positive definitive operator $\underset{x 0}{D^{2}}=-\frac{\mathrm{d}^{2}}{\mathrm{~d} x^{2}}$ and must not be confused with a power of the first order differential operator $\underset{x 0}{D^{1}}$ (see [4] for a detailed theory of this operator and related operators). I need to adopt the Fourier transform of a (generalized) function $f(x), x \in \mathbb{R}$, which is defined as

$$
\mathcal{F}\{f(x) ; \kappa\}=\hat{f}(\kappa)=\int_{-\infty}^{\infty} \mathrm{e}^{i \kappa x} f(x) \mathrm{d} x .
$$

For the proof of convergence in distribution I need to use the Fourier transform of $\underset{x 0}{D^{\alpha}}$ which reads

$$
\mathcal{F}\left\{D_{0}^{\alpha} \Phi(x) ; \kappa\right\}=-|\kappa|^{\alpha} \hat{\phi}(\kappa), 0<\alpha \leq 2, \kappa \in \mathbb{R},
$$

while

$$
\mathcal{F}\left\{\frac{\mathrm{d}^{n}}{\mathrm{~d} x^{n}} \Phi(x) ; \kappa\right\}=-(i \kappa)^{n} \hat{\phi}(\kappa), n \in \mathbb{N}, \kappa \in \mathbb{R}
$$

This means, in the Zaslavski' s notations,

$$
D_{0}^{\alpha} \Phi(x)=\frac{\mathrm{d}^{\alpha}}{\mathrm{d}|x|^{\alpha}}, 0<\alpha \leq 2 .
$$

From (2.2) - (2.4), one easily sees that in the case $\alpha=1$

$$
\mathcal{F}\left\{D_{0}^{1} \phi(x) ; \kappa\right\} \neq \mathcal{F}\left\{\frac{\mathrm{d} \phi(x)}{\mathrm{d} x} ; \kappa\right\} .
$$

Since $-|\kappa|^{\alpha}=-\left(\kappa^{2}\right)^{\alpha / 2}$, we can set $D_{x 0}^{\alpha}=-\left(\frac{\mathrm{d}^{2}}{\mathrm{~d} x^{2}}\right)^{\alpha / 2}$, 
which proves that the Riesz derivative is a symmetric fractional generalization of the second derivative. For more information about the Fourier transform and the pseudo-differential operators as semi groups of linear operators, see e.g. [26,27]. In my paper, I discuss the approximate solution of the Equation (2.1) for all values of $\alpha$ and $\beta$, to do so, I descretize $x$ and $t$ by the grid $\left\{\left(x_{j}, t_{n}\right) \mid-M \leq j \leq M, n \geq 0\right\}$ with $x_{j}=j h$,

$t_{n}=n \tau$. Here $h>0$, and $\tau>0$ are the steps in space and in time, respectively, and $N=2 M+1$ is the number of steps at the $x$ direction. Treating $u(x, t)$ as a density of an extensive quantity (like mass, charge, solute concentration, or probability), the approximation of the collected quantity $\int_{x_{j}-\frac{h}{2}}^{x_{j}+\frac{h}{2}} u\left(x, t_{n}\right) \mathrm{d} x$ presents in a spatial cell $x_{j}-\frac{h}{2}<x \leq x_{j}+\frac{h}{2}$ at the instant $t=t_{n}$ by a clump $y_{j}^{(n)}$,

$$
y_{j}^{(n)} \approx \int_{x_{j}-\frac{h}{2}}^{x_{j}+\frac{h}{2}} u\left(x, t_{n}\right) \mathrm{d} x .
$$

For $-M \leq j \leq M$, I introduce the column vector

$$
y^{(n)}=\left\{y_{-M}^{(n)}, y_{-M+1}^{(n)}, \cdots, 0, \cdots, y_{M-1}^{(n)}, y_{M}^{(n)}\right\}^{\mathrm{T}},
$$

where $y^{(n)}=y\left(t_{n}\right)$. To proceed on the proof of convergence in distribution, one needs to use the method of generating functions, see [22] for more information about the procedures used to prove the convergence. Therefore, for $n \in(0, \infty]$, define

$$
q_{n}(z)=\sum_{j} y_{j}\left(t_{n}\right) z^{j},
$$

for the sequence of clumps

$\left\{y_{-M}^{(n)}, y_{-M+1}^{(n)}, \cdots, 0, \cdots, y_{M-1}^{(n)}, y_{M}^{(n)}\right\}$. Using the initial conditions, I introduce the function

$$
\sum_{j} \delta\left(x-x_{j}\right) y_{j}\left(t_{n}\right), \forall n \geq 0,
$$

and applying the Fourier-transform, we obtain

$$
\begin{aligned}
& \mathcal{F}\left\{\sum_{j} \delta\left(x-x_{j}\right) y_{j}\left(t_{n}\right) ; \kappa\right\}=\sum_{j} \mathrm{e}^{i \kappa x_{j}} y_{j}\left(t_{n}\right)=q_{n}\left(\mathrm{e}^{i \kappa h}\right), \\
& \kappa \in \mathbb{R} .
\end{aligned}
$$

Now, introduce the bivariate (two-fold) generating function

$$
Q(z, \zeta)=\sum_{n} q_{n}(z) \zeta^{n}=\sum_{n}\left(\sum_{j} y_{j}\left(t_{n}\right) z^{j}\right) \zeta^{n},
$$

as a function of $\zeta$, where $|\zeta| \leq 1$, for the sequence

$$
\left\{q_{-M}(z), q_{-M+1}(z), \cdots, 0, \cdots, q_{M-1}(z), q_{M}(z)\right\} .
$$

Introduce the function $\sum_{n=0}^{\infty} \delta\left(t-t_{n}\right) q_{n}(z)$ and apply the Laplace-transform, one gets

$$
\mathcal{L}\left\{\sum_{n} \delta\left(t-t_{n}\right) q_{n}(z) ; s\right\}=\sum_{n} \mathrm{e}^{-s t_{n}} q_{n}(z), s>0 .
$$

From Equations (2.7) and (2.10), we deduce that if we replace $z$ by $\mathrm{e}^{i \kappa h}$ and $\zeta$ by $\mathrm{e}^{-s \tau}$, in Equation (2.8), we get the Fourier-Laplace transform of the bivariate sequence $\left(y_{j}\left(t_{n} \mid j \in \mathbb{Z}, n \in \mathbb{N}_{0}\right)\right)$ which is obtained by collecting all the sequences in (2.9). This means

$$
Q\left(\mathrm{e}^{i \kappa h}, \mathrm{e}^{-s \tau}\right)=\sum_{n=0}^{\infty}\left(\sum_{j} y_{j}\left(t_{n}\right) \mathrm{e}^{i \kappa j h}\right) \mathrm{e}^{-n s \tau}, \kappa \in \mathbb{R}, s>0 .
$$

Our aim now is to prove that $Q\left(\mathrm{e}^{i \kappa h}, \mathrm{e}^{-s \tau}\right)$ is related asymptotically to the Fourier-Laplace transform of $u(x, t)$ which represents the analytical solution of Equation (2.1) for any values of $\alpha$ and $\beta$, and for a fixed $\kappa \in \mathbb{R}$ and $s>0$, as $n \rightarrow \infty$. So far, I will prove

$$
\lim _{h, \tau \rightarrow 0} \tau Q\left(\mathrm{e}^{i \kappa h}, \mathrm{e}^{-s \tau}\right)=\hat{\tilde{u}}(\kappa, s),
$$

for each case.

\section{The Classical ade}

I describe in this section the classical partial differential ade and its proof of convergence in distribution. It is well known that the classical ade is a partial differential equation describing the solute transport in aquifers and it reads

$$
\frac{\partial u(x, t)}{\partial t}=a \frac{\partial^{2} u(x, t)}{\partial x^{2}}-b \frac{\partial u(x, t)}{\partial x},
$$

here $u(x, t)$ is the solute concentration. The conditions imposed on the solution $u(x, t)$ are

$$
u(x, t) \geq 0 \text { and } \int_{-\infty}^{\infty} u(x, t)=1 .
$$

With the initial condition $u(x, 0)=\delta\left(x-x_{0}\right)$. The classical ade is also interpreted as a deterministic equation with the probability function $u(x, t)$ which describes the particle spreading away from the plume center of mass. The stochastic process $\{X(t): t \geq 0\}$ described by Equation (3.1) is a Brownian motion with a constant drift [11].

If $b=0$, then one has the diffusion of a free particle, that is, a particle in which no forces other than those due to the molecules of the surrounding medium are acting, which reads $\frac{\partial v(\zeta, \tau)}{\partial \tau}=a \frac{\partial^{2} v(\zeta, \tau)}{\partial \zeta^{2}}$. It has the solution 
$v(\zeta, \tau)=\frac{1}{2 \sqrt{\pi a \tau}} \mathrm{e}^{-\zeta^{2} /(4 a \tau)}$. Consequently by using the Galilei transformation of the independent variables $(\zeta, \tau)$ to $(x-b t, t)$, then the solution of (3.1), as $b \neq 0$, is $u(x, t)=\frac{1}{2 \sqrt{\pi a t}} \mathrm{e}^{-(x-b t)^{2} /(4 t)}$. In the Fourier domain $\hat{u}(\kappa, t)=\mathrm{e}^{-i b \kappa t} \hat{v}(\kappa, t)$ and hence fourth in the FourierLaplace domain, see [28]

$$
\hat{\tilde{u}}(\kappa, s)=\hat{\tilde{v}}(s+i b \kappa, s)=\frac{1}{s+a|\kappa|^{2}-i b \kappa} .
$$

Now descretizing (3.1) by the central symmetric difference in space and forward in time, one gets

$$
\frac{y_{j}^{(n+1)}-y_{j}^{(n)}}{\tau}=a \frac{y_{j+1}^{(n)}-2 y_{j}^{(n)}+y_{j-1}^{(n)}}{h^{2}}-\frac{b}{2 h}\left(y_{j+1}^{(n)}-y_{j-1}^{(n)}\right) .(3.3)
$$

Introduce the scaling relation

$$
\mu=\frac{\tau}{h^{2}},
$$

and for the positivity of all the coefficients of $y_{j}^{(n)}$, one must put $0 \leq \mu \leq 1 / 2$. Now let $N=2 / h$, one can write $y_{j}^{(n+1)}$ as

$$
y_{j}^{(n+1)}=\mu y_{j-1}^{(n)}\left(a+\frac{b}{N}\right)+y_{j}^{(n)}(1-2 a \mu)+\mu y_{j+1}^{(n)}\left(a-\frac{b}{N}\right) .
$$

The discrete solution at Equation (3.5) describes also a random walk with sojourn probability $y_{j}^{(n)}$ of a particle at the point $x_{j}$ at the instant $t_{n}$ and it may jump either to the points $x_{j-1}, x_{j}$, or $x_{j+1}$ at the time instant $t_{n+1}$, see [29]. Utilizing this concept, Equation (3.5) can be rewritten as

$$
y_{j}^{(n+1)}=p_{j j-1} y_{j-1}^{(n)}+p_{j j} y_{j}^{(n)}+p_{j j+1} y_{j+1}^{(n)},
$$

The transition probabilities $p_{j j-1}, p_{j j}$ and $p_{i j+1}$ in Equation (3.6) satisfy the essential condition

$$
p_{j j-1}+p_{j j}+p_{j j+1}=1,-M \leq j \leq M, N=2 M+1 .
$$

Now one can use these transition probabilities to constitute a tridiagonal, $P$ matrix, in which

$p_{i j}=0, \forall|i-j| \geq 2$. Therefore, Equation (3.5) is written in the following matrix form

$$
y^{(n+1)}=P^{\mathrm{T}} \cdot y^{(n)},
$$

Introduce the row vector $z^{(n)}$, defined as

$$
\begin{aligned}
& \left(y^{(n)}\right)^{\mathrm{T}}=z^{(n)}, \forall n \geq 0, z^{(n)} \\
& =\left\{z_{-M}^{(n)}, z_{-M+1}^{(n)}, \cdots, 0, \cdots, z_{M-1}^{(n)}, z_{M}^{(n)}\right\} .
\end{aligned}
$$

In order to find the explicit discrete solution of Equation (3.1), I have to take the transpose of each sides of the matrix Equation (3.7) and rewrite it as

$$
z^{(n+1)}=z^{(n)} \cdot P,
$$

and for the numerical calculations, it is convenient to write the stochastic matrix $P$ in the form

$$
P=(I+\mu H),
$$

here $I$ is the unit matrix and $H$ is a $N \times N$ matrix whose rows are summed to zero. In Section 7, I give the evolution of $y\left(t_{n}\right)=y^{(n)}$ for different values of $t$. Now I am going to prove that the discrete solution at Equation (3.5) converges to the Fourier-Laplace transform of Equation (3.1). Rewrite Equation (3.5) as

$$
y_{j}^{(n+1)}-y_{j}^{(n)}=\mu y_{j-1}^{(n)}\left(a+\frac{b}{N}\right)-2 a \mu y_{j}^{(n)}+\mu y_{j+1}^{(n)}\left(a-\frac{b}{N}\right) .
$$

Then multiplying both sides by $z^{j}$ and summing over all $j$, to get

$$
q_{n+1}(z)-q_{n}(z)=\mu q_{n}(z)\left\{\frac{1}{z}\left(a-\frac{b}{N}\right)+z\left(a+\frac{b}{N}\right)-2 a\right\}
$$

Multiplying both sides by $\zeta^{n}$ and summing over all $n \in \mathbb{N}_{0}$, one gets

$$
\begin{aligned}
& \sum_{n=0}\left(q_{n+1}-q_{n}\right) \zeta^{n}-q_{0}(z) \\
= & \mu Q(z, \zeta)\left\{\frac{1}{z}\left(a-\frac{b}{N}\right)+z\left(a+\frac{b}{N}\right)-2 a\right\} .
\end{aligned}
$$

The choice of the initial condition of the column vector $y^{(0)}$ satisfying that $\sum_{j} y_{j}^{(0)}=1$, guarantees that $q_{0}(z)=1$

$$
\begin{aligned}
& Q(z, \zeta)\left(\frac{1}{\zeta}-1\right)-1 \\
= & \mu Q(z, \zeta)\left\{\frac{1}{z}\left(a-\frac{b}{N}\right)+z\left(a+\frac{b}{N}\right)-2 a\right\} .
\end{aligned}
$$

Now, replace $z$ by $\mathrm{e}^{i \kappa h}$ and $\zeta$ by $\mathrm{e}^{-s \tau}$, in Equation (3.12), to get

$$
\begin{aligned}
& \left(\frac{1}{\mathrm{e}^{-s \tau}}-1\right) Q\left(\mathrm{e}^{i \kappa h}, \mathrm{e}^{-s \tau}\right)-1 \\
& =\mu Q\left(\mathrm{e}^{i \kappa h}, \mathrm{e}^{-s \tau}\right)\left\{\mathrm{e}^{-i \kappa h}\left(a-\frac{b}{N}\right)+\mathrm{e}^{i \kappa h}\left(a+\frac{b}{N}\right)-2\right\} a
\end{aligned}
$$

Now after using Taylor expansion and taking the limits as $h \rightarrow 0$ and $\tau \rightarrow 0$, one gets

$$
\tau Q\left(\mathrm{e}^{i \kappa h}, \mathrm{e}^{-s \tau}\right)=\frac{1}{s-i b \kappa+a|\kappa|^{2}} .
$$

Compare this equation with Equation (3.2), then Equa- 
tion (2.12), is satisfied for the classical ade.

\section{The Time-Fractional ade}

In this section, I replace the first-order time derivative in Equation (3.1) by the Caputo fractional derivative, $D_{t^{*}}^{\beta} u(x, t)=\frac{\partial^{\beta} u(x, t)}{\partial t^{\beta}} u(x, t)$, with $\beta \in(0,1]$. Then this generalized time-fractional advection-dispersion equation, fade, reads

$$
\begin{aligned}
& \frac{\partial^{\beta} u(x, t)}{\partial t^{\beta}}=a \frac{\partial^{2} u(x, t)}{\partial x^{2}}-b \frac{\partial}{\partial x} u(x, t), \\
& 0<\beta \leq 1, u(x, 0)=\delta(x) .
\end{aligned}
$$

For more information about the Caputo fractional derivative and its relations to the Riemann-Liouville, see [6] and the list of references therein. Now, Taking the Fourier-Laplace transform, see Section 2, one gets

$$
\hat{\tilde{u}}(\kappa, s)=\frac{s^{\beta-1}}{s^{\beta}+a|\kappa|^{2}-i b \kappa} .
$$

To descretize $D^{\beta} u(x, t)$, I utilize the backward Grünwald-Letnikov scheme which has been successfully utilizing at [19-24] for modelling and simulating the timefractional diffusion processes and the time-fractional Fokker-Planck equations.

$$
{ }_{\tau^{*}}{ }^{\beta} y_{j}^{(n+1)}=\sum_{m=0}^{n+1}(-1)^{m}\left(\begin{array}{c}
\beta \\
m
\end{array}\right) \frac{y_{j}^{(n+1-m)}-y_{j}^{(0)}}{\tau^{\beta}}, 0<\beta \leq 1 .
$$

Join this discretization with the common symmetric finite difference for $\frac{\partial^{2} u(x, t)}{\partial x^{2}}$ and $\frac{\partial}{\partial x} u(x, t)$, then one has

$$
\begin{aligned}
& \tau^{-\beta} \sum_{m=0}^{n+1}(-1)^{m}\left(\begin{array}{c}
\beta \\
m
\end{array}\right)\left(y_{j}^{(n+1-m)}-y_{j}^{(0)}\right) \\
& =\frac{a}{h^{2}}\left(y_{j+1}^{(n)}-2 y_{j}^{(n)}+y_{j-1}^{(n)}\right)-\frac{b}{2 h}\left(y_{j+1}^{(n)}-y_{j-1}^{(n)}\right) .
\end{aligned}
$$

Now introduce the scaling parameter

$$
\mu=\frac{\tau^{\beta}}{h^{2}},
$$

and solve for $y_{j}^{(n+1)}$, one gets

$$
\begin{aligned}
y_{j}^{(n+1)}= & b_{n} y_{j}^{(0)}+\sum_{m=2}^{n} c_{m} y_{j}^{(n+1-m)}+(\beta-2 a \mu) y_{j}^{(n)} \\
& +\mu\left(a-\frac{b}{N}\right) y_{j+1}^{(n)}+\mu\left(a+\frac{b}{N}\right) y_{j-1}^{(n)},
\end{aligned}
$$

where for ease of writing, I use $b_{n}$ and $c_{m}$ which has been originally introduced in [19] as

$$
\begin{gathered}
b_{n}=\sum_{m=n+1}^{\infty}(-1)^{m}\left(\begin{array}{l}
\beta \\
m
\end{array}\right), \\
c_{m}=(-1)^{m+1}\left(\begin{array}{l}
\beta \\
m
\end{array}\right), m=1,2, \cdots
\end{gathered}
$$

with $b_{0}=c_{1}=\beta$, and all $c_{k} \geq 0, b_{n} \geq 0$. Finally, $b_{n}$ and $c_{m}$ satisfy the relation

$$
b_{n}+\sum_{m=1}^{n} c_{m}=1
$$

where $c_{0}=1$, see [19]. For all the coefficients of $y_{j}^{(n)}$ to be positive, it is required that $0<\mu \leq \frac{\beta}{2}$. Rewrite Equation (4.6) in the following form

$$
\begin{aligned}
& y_{j}^{(n+1)} \\
= & \left(1-\sum_{m=1}^{n} c_{m}\right) y_{j}^{(0)}+c_{n} y_{j}^{(1)}+c_{n-1} y_{j}^{(2)}+\cdots+c_{2} y_{j}^{n-1} \\
& +\left(c_{1}-2 a \mu\right) y_{j}^{(n)}+\mu\left(a-\frac{b}{N}\right) y_{j+1}^{(n)}+\mu\left(a+\frac{b}{N}\right) y_{j-1}^{(n)} .
\end{aligned}
$$

This equation can be interpreted as a random walk with a memory, see [30]. In this case, the particle is sitting at the position $x_{j}$ at the time instant $t_{n}$ and can move to either $x_{j-1}, x_{j}$, or $x_{j+1}$ at the time instant $t_{n+1}$. It has also the possibility to return back to $x_{j}$ at any of the time instants $\left\{t_{n-1}, t_{n-2}, \cdots, t_{0}\right\}$. By using the identity (4.7), it is easily to prove that the summation of the transition probabilities at the right hand side of Equation (4.8) equals to one. These transitions constitute a symmetric random walk. Now, constitute the column vector $y^{(n+1)}=\left\{y_{-M}^{(n+1)}, y_{-M+1}^{(n+1)}, \cdots, 0, \cdots, y_{M-1}^{(n+1)}, y_{M}^{(n+1)}\right\}$, then Equation (4.6) is written in the matrix form

$$
y^{(n+1)}=b_{n} y^{(0)}+\sum_{m=2}^{n} c_{m} y_{j}^{(n+1-m)}+Q^{\mathrm{T}} \cdot y^{(n)},
$$

where $Q$ is $N \times N$ matrix and is not a stochastic matrix as its rows are summed to $\beta$, where $0<\beta<1$. A useful numerical method to ease the computation is to write the matrix $Q$ as

$$
Q=\beta I+\mu H,
$$

where $H$ is the same matrix defined in the last section, i.e. it does not depend on the value of $\beta$ then it does not depend on the time. In Section 7, I compare the evolution of $y^{(n)}$ for different values of $t$ and $\beta$.

Now, I want to prove that the discrete solution (4.6), in the Fourier-Laplace domain as $h \rightarrow 0$ and $\tau \rightarrow 0$, converges to the Fourier-Laplace transform of Equation (4.1). To do so, I have to adopt the initial condition which satisfies that $\sum_{j} y_{j}^{(0)}=1$, then $q_{0}(z)=\hat{\delta}(\kappa)=1$. Then 
multiply each sides of Equation (4.4) by $z^{j}$ and sum over all $j$, to get

$$
\begin{aligned}
& \sum_{m=0}^{n+1}(-1)^{m}\left(\begin{array}{c}
\beta \\
m
\end{array}\right)\left(q_{n+1-m}(z)-1\right) \\
= & a \mu q_{n}(z)\left(\frac{1}{z}-2+z\right)-\frac{b \tau^{-\beta}}{2 h} q_{n}(z)\left(\frac{1}{z}-z\right) .
\end{aligned}
$$

Now proceed further, multiply each sides by $\zeta^{m}$ and sum over all $m$, to get

$$
\begin{aligned}
& \sum_{n=0}^{\infty} \sum_{m=0}^{n+1}(-1)^{m}\left(\begin{array}{c}
\beta \\
m
\end{array}\right)\left(q_{n+1-m}(z)-1\right) \zeta^{m} \\
= & a \mu Q(z, \zeta)\left\{\left(\frac{1}{z}-2+z\right)-\frac{b \tau^{-\beta}}{2 h}\left(\frac{1}{z}-z\right)\right\} .
\end{aligned}
$$

For manipulating the R.H.S., one needs to use the rule of multiplication of two sequences $\alpha(z)=\sum_{n=0}^{\infty} \alpha_{n} \zeta^{n}$ and $\beta(\zeta)=\sum_{k=0}^{\infty} \beta_{k} \zeta^{k}$, see Feller [29], in which

$$
\alpha(\zeta) \cdot \beta(\zeta)=\sum_{r=0}^{\infty} c_{r} \zeta^{r}, c_{r}=\sum_{n=0}^{r} \alpha_{n} \beta_{r-n} .
$$

After using this rule, and put $z=\mathrm{e}^{-i \kappa h}$, then apply Taylor expansion, and take the limit as $h \rightarrow 0$, one gets

$$
\begin{aligned}
& (1-\zeta)^{\beta}\left(Q\left(\mathrm{e}^{-i \kappa h}, \zeta\right)-\frac{1}{1-\zeta}\right) \\
= & Q\left(\mathrm{e}^{-i \kappa h}, \zeta\right)\left(-a \mu \kappa^{2} h^{2}+2 i b \kappa h \frac{\tau^{-\beta}}{2 h}\right)
\end{aligned}
$$

Now, substitute $\zeta=\mathrm{e}^{-s \tau}$, then again apply Taylor expansion, and take the limit as $\tau \rightarrow 0$, you get

$$
Q\left(\mathrm{e}^{-i \kappa h}, \mathrm{e}^{-s \tau}\right)=\frac{s^{\beta-1} \tau^{\beta-1}}{s^{\beta} \tau^{\beta}+a \kappa^{2} \tau^{\beta}-i b \kappa \tau^{\beta}},
$$

then multiply each sides by $\tau$, one gets

$$
\tau Q\left(\mathrm{e}^{-i \kappa h}, \mathrm{e}^{-s \tau}\right)=\hat{\tilde{u}}(\kappa, s) .
$$

Then I have proved the required aim for the timefractional ade.

\section{The Space-Time-Fractional ade}

In this section I consider the space-time fade, Equation (2.1). It is known that the space-fractional ade arises when velocity variations are heavy tailed and describe particle motion that accounts for variation in the flow field over entire system. The time fractional ade arises as a result of power law particle residence time distributions and describe particle motion with memory in time, see [12]. The used space-fractional operator $\underset{x 0}{D^{\alpha}}$, is the sym- metric Feller operator, see [7]. This operator represents the negative inverse of the Riesz Potential $I_{0}^{\alpha}$ whose symbol is $|\kappa|^{\alpha}$, i.e.

$$
\underset{x}{D^{\alpha}}=-I_{0}^{-\alpha},
$$

where the symmetric Riesz Potential operator is defined as

$$
I_{0}^{\alpha} \Phi(x)=c_{-}(\alpha) I_{+}^{\alpha} \Phi(x)+c_{+}(\alpha) I_{-}^{\alpha} \Phi(x), \alpha \neq 1,
$$

where

$$
c_{+}(\alpha)=c_{-}(\alpha)=1 /\left(2 \cos \frac{\alpha \pi}{2}\right) .
$$

The Fourier-Laplace transformation of Equation (2.1) reads

$$
\hat{\tilde{u}}(\kappa, s)=\frac{s^{\beta-1}}{s^{\beta}+a|\kappa|^{\alpha}-i b \kappa} .
$$

When descretizing the Riesz fractional operator $\underset{x 0}{D^{\alpha}}$ one must use a suitable finite difference scheme and exclude the case $\alpha=1$. To do so, I use the approximation of the inverse operators $I_{ \pm}^{-\alpha}$ by the Grünwald-Letnikov scheme, see Oldham \& Spanier [31], Ross \& Miller [1] and see also [6], in which one can find a long list of related references. The inverse of the Riemann-Liouville integrals can formally be obtained as the limit

$$
I_{ \pm}^{-\alpha}=\lim _{h \rightarrow 0 h \pm} I^{-\alpha},
$$

where $I^{-\alpha}$ denotes the approximating Grünwald-Letnikov scheme which reads, see $[4,18,19]$

a) $0<\alpha<1$

$$
I_{ \pm}^{-\alpha} \phi(x)=\frac{1}{h^{\alpha}} \sum_{k=0}^{\infty}(-1)^{k}\left(\begin{array}{l}
\alpha \\
k
\end{array}\right) \phi\left(x_{j} \mp k h\right),
$$

b) $1<\alpha \leq 2$

$$
I_{ \pm}^{-\alpha} \phi(x)=\frac{1}{h^{\alpha}} \sum_{k=0}^{\infty}(-1)^{k}\left(\begin{array}{l}
\alpha \\
k
\end{array}\right) \phi\left(x_{j} \mp(k-1) h\right) .
$$

The shift in the index $j$ in Equation (5.5) is required to obtain a scheme with all coefficients are non-negative in the final formula for $y_{j}\left(t_{n+1}\right)$ which gives schemes for simulating particle paths which results after replacing the second order space-derivative in Equation (4.1) by the Feller operator [7]. One can adopt, for simplicity, the notation introduced by Zaslavski [25].

$$
\begin{aligned}
& D_{h 0}^{\alpha} y_{j}\left(t_{n}\right)=\frac{-1}{2 \cos \frac{\alpha \pi}{2}}\left(I_{h+}^{I^{-\alpha}}+I_{h-}^{-\alpha}\right) y_{j}\left(t_{n}\right), \\
& 0<\alpha \leq 2, \alpha \neq 1, j \in \mathbb{Z} .
\end{aligned}
$$

one must distinguish the descretization of $I_{h \pm}^{-\alpha}$ with 
respect to the value of $\alpha$, as follows:

$$
I_{h \pm}^{-\alpha} y_{j}\left(t_{n}\right)=\frac{1}{h^{\alpha}} \sum_{k=0}^{\infty}(-1)^{k}\left(\begin{array}{l}
\alpha \\
k
\end{array}\right) y_{j \mp k}, 0<\alpha \leq 1,
$$

while

$$
I_{h \pm}^{-\alpha} y_{j}\left(t_{n}\right)=\frac{1}{h^{\alpha}} \sum_{k=0}^{\infty}(-1)^{k}\left(\begin{array}{l}
\alpha \\
k
\end{array}\right) y_{j \pm 1 \mp k}, 1<\alpha \leq 2,
$$

Now we adjoin the descretization of $D_{h 0}^{\alpha}$, with the descretization of $D_{\tau^{*}}^{\beta}$, and with the finite sequence $\left\{y_{j}\left(t_{n}\right)\right\}$. In what follows, I give the descretization of the space-time fractional ade for each case.

\subsection{Case (a): $0<\alpha<1, \quad 0<\beta \leq 1$}

In order to ensure that all the coefficients of $y_{j}^{(n)}>0 \forall j$, I have to descritize $\frac{\partial^{2} u(x, t)}{\partial x^{2}}$ by using the symmetric central scheme, so the discretization of Equation (2.1) reads

$$
\begin{aligned}
& \tau^{-\beta} \sum_{m=0}^{n+1}(-1)^{m}\left(\begin{array}{l}
\beta \\
m
\end{array}\right)\left(y_{j}^{(n+1-m)}-y_{j}^{(0)}\right) \\
= & -a h^{-\alpha} \sum_{k=0}^{N}(-1)^{k}\left(\begin{array}{l}
\alpha \\
k
\end{array}\right)\left(c_{+} y_{j-k}^{(n)}+c_{-} y_{j+k}^{(n)}\right) \\
& -\frac{b}{2 h}\left(y_{j+1}^{(n)}-y_{j-1}^{(n)}\right) .
\end{aligned}
$$

Adopting the scaling relation

$$
\mu=\frac{\tau^{\beta}}{h^{\alpha}},
$$

and using Equation (5.1), one can solve Equation (5.9) for $y_{j}^{(n+1)}$ to get

$$
\begin{aligned}
y_{j}^{(n+1)}= & b_{n} y_{j}^{(0)}+\sum_{m=2}^{n} c_{m} y_{j}^{(n+1-m)}+\left(\beta-\frac{a \mu}{\cos \frac{\alpha \pi}{2}}\right) y_{j}^{(n)} \\
& +\left(\frac{a \mu}{2 \cos \frac{\alpha \pi}{2}}\left(\begin{array}{l}
\alpha \\
1
\end{array}\right)+\frac{b \tau^{\beta}}{2 h}\right) y_{j-1}^{(n)} \\
& +\left(\frac{a \mu}{2 \cos \frac{\alpha \pi}{2}}\left(\begin{array}{l}
\alpha \\
1
\end{array}\right)-\frac{b \tau^{\beta}}{2 h}\right) y_{j+1}^{(n)} \\
& +\frac{a \mu}{2 \cos \frac{\alpha \pi}{2}}\left(\sum_{k \geq 2}^{N}(-1)^{k+1}\left(\begin{array}{l}
\alpha \\
k
\end{array}\right)\left(y_{j-k}^{(n)}+y_{j+k}^{(n)}\right)\right) .
\end{aligned}
$$

To ensure that the coefficients of all $y_{j}^{(n)} \geq 0$, it requires that

$$
0 \leq \mu \leq \frac{\beta}{a} \cos \frac{\alpha \pi}{2} .
$$

Let us write Equation (5.11) in the form of a random walk, in which the walker is sitting at $x_{j}$ at $t_{n}$ and jumps to $x_{k}$ at $t_{n+1}$, where

$$
\begin{aligned}
& y_{k}^{(n+1)}=\sum_{j=-\infty}^{\infty} p_{j, k} y_{j}^{(n)}, p_{j, k}=p_{k, j} \\
& \text { and } \sum_{j=-\infty}^{\infty} p_{j, k}=\sum_{k=-\infty}^{\infty} p_{j, k}=1,
\end{aligned}
$$

see [32] for more information about the discrete random walk of space-fractional diffusion processes. Then by using this notations, Equation (5.11) can be written in the form of random walk as

$$
\begin{aligned}
y_{j}^{(n+1)}= & b_{n} y_{j}^{(0)}+\sum_{m=2}^{n} c_{m} y_{j}^{(n+1-m)}+p_{j, j} y_{j}^{(n)} \\
& +p_{j, j-1} y_{j-1}^{(n)}+p_{j, j+1} y_{j+1}^{(n)} \\
& +\sum_{k \geq 2}^{N}\left(p_{j, j-k} y_{j-k}^{(n)}+p_{j, j+k} y_{j+k}^{(n)}\right) .
\end{aligned}
$$

The first two terms at the LHS of this equation represent the memory part and the other terms represent the diffusion under the drift term. I like to write $p_{j, j}=p_{0}$ as it represents the transition to the next step at the same point, $p_{j, j-1}=p_{-1}$ represents jumping one step to the left, $p_{+1}=p_{j, j+1}$ represents jumping one step to the right, and similarly $p_{ \pm k}=p_{j \pm k}, k \geq 2$ jumping $k$ steps to the left or right. By using the identity (4.7), and the identity

$$
\sum_{k=-\infty}^{\infty}(-1)^{k}\left(\begin{array}{l}
\alpha \\
k
\end{array}\right)=0
$$

then it is easily to prove that the summation of all the transition probabilities is one.

Now for the numerical calculations, and since there is a symmetric random walk, I adjust $j \in[-M, M]$ and ignore all the transitions outside this interval. So it is convenient to write the last equation in the form of a matrix form

$$
\begin{aligned}
y^{(n+1)}= & b_{n} y^{(0)}+\sum_{m=2}^{n} c_{m} y_{j}^{(n+1-m)} \\
& +A^{\mathrm{T}} \cdot y^{(n)} .
\end{aligned}
$$

Here $A=a_{i j}$ is an elegant $N \times N$ fifth diagonal matrix with its elements are computed as 


$$
a_{i j}= \begin{cases}a_{i j}^{(1)}=p_{+k}=\frac{a \mu}{2 \cos \frac{\alpha \pi}{2}}(-1)^{j-i+1}\left(\begin{array}{c}
\alpha \\
|j-i|
\end{array}\right) & j \geq i+2, i=-M+3, \cdots, M-3 \\
a_{i j}^{(2)}=p_{+1}=\frac{a \mu}{2 \cos \frac{\alpha \pi}{2}}\left(\begin{array}{c}
\alpha \\
1
\end{array}\right)-\frac{b \tau^{\beta}}{2 h} & j=i+1, i=-M, \cdots, M-2 \\
a_{i j}^{(3)}=p_{0}=\beta-\frac{a \mu}{\cos \frac{\alpha \pi}{2}} & j=i=-M,-M+1, \cdots, M \\
a_{i j}^{(4)}=p_{-1}=\frac{a \mu}{2 \cos \frac{\alpha \pi}{2}}\left(\begin{array}{c}
\alpha \\
1
\end{array}\right)+\frac{b \tau^{\beta}}{2 h} \quad j=i-1, i=-M+1,-M+2, \cdots, M-1 \\
a_{i j}^{(5)}=p_{-k}=\frac{a \mu}{2 \cos \frac{\alpha \pi}{2}}(-1)^{j-i+1}\left(\begin{array}{c}
\alpha \\
|i-j|
\end{array}\right) & j \leq i-2, i=-M+3, \cdots, M-1,\end{cases}
$$

where $|j-i|=k$ and $i, j \in[-M, M]$. In the special case as $\alpha=2$, one recovers the well-known three point jumps of the classical ade because $p_{j, j \pm k}=p_{ \pm k}=0$ as $k \geq 2$, and hence the matrix $A$ be the same as matrix $p$ defined in Equation (3.7). In Section 7, I will plot the path of the particle representing by (5.13). Now, I will prove that the discrete solution at (5.9) converges in the Fourier-Laplace domain to the Fourier-Laplace transform of Equation (2.1) as $0 \leq \alpha<1$. To do so, multiplying both sides of Equation (5.9) by $z_{j}$ where and sum over all $j$, where it is easy to prove

$\sum_{j} \sum_{k}(-1)^{k}\left(\begin{array}{l}\alpha \\ k\end{array}\right)\left(y_{j-k}^{(n)}+y_{j+k}^{(n)}\right)=q_{n}(z)\left(\left(1-\frac{1}{z}\right)^{\alpha}+(1-z)^{\alpha}\right)$,

then one gets

$$
\begin{aligned}
& \sum_{m=0}^{n+1}(-1)^{m}\left(\begin{array}{l}
\beta \\
m
\end{array}\right)\left(q_{n+1-m}(z)-1\right) \\
= & -\frac{a \mu q_{n}(z)}{2 \cos \frac{\alpha \pi}{2}}\left(\left(1-\frac{1}{z}\right)^{\alpha}+(1-z)^{\alpha}\right)-\frac{b \tau^{\beta}}{2 h}\left(\frac{1}{z}-z\right) q_{n}(z) .
\end{aligned}
$$

The second step is to multiply both sides of this equation by $\zeta^{n}$ and sum over all $n$,

$$
\begin{aligned}
(1-\zeta)^{\beta} & \left(Q(z, \zeta)-\frac{1}{1-\zeta}\right) \\
=Q(z, \zeta) & \left\{-\frac{a \zeta \mu}{2 \cos \frac{\alpha \pi}{2}}\left(\left(1-\frac{1}{z}\right)^{\alpha}+(1-z)^{\alpha}\right)\right. \\
& \left.-\frac{b \zeta \tau^{\beta}}{2 h}\left(\frac{1}{z}-z\right)\right\} .
\end{aligned}
$$

then put $z=\mathrm{e}^{i \kappa h}$ and $\zeta=\mathrm{e}^{-s \tau}$ and use the previous results. The only new part on the proof is as $h \rightarrow 0$, one can easily prove by using the fundamentals of complex analysis that

$$
\begin{aligned}
& \left(1-\mathrm{e}^{-i \kappa h}\right)^{\alpha}+\left(1-\mathrm{e}^{i \kappa h}\right)^{\alpha}=\mathrm{e}^{-\frac{i \pi \alpha}{2}}(\kappa h)^{\alpha}+\mathrm{e}^{\frac{i \pi \alpha}{2}}(\kappa h)^{\alpha} \\
& =2(\kappa h)^{\alpha} \cos \frac{\alpha \pi}{2} .
\end{aligned}
$$

So far

$$
Q\left(\mathrm{e}^{i \kappa h}, \mathrm{e}^{-s \tau}\right)=\frac{(s \tau)^{\beta-1}}{(s \tau)^{\beta}+a \mu|h \kappa|^{\alpha}-i b \kappa \tau^{\beta}} .
$$

Finally multiply each side by $\tau$ and substitute the value of $\mu$, and compare with (5.2), one gets

$$
\tau Q\left(\mathrm{e}^{i \kappa h}, \mathrm{e}^{-s \tau}\right)=\hat{\tilde{u}}(\kappa, s) .
$$

So, I proved the desired aim.

\subsection{Case (b): $1<\alpha<2, \quad 0<\beta \leq 1$}

I have to descritize $\frac{\partial^{2} u(x, t)}{\partial x^{2}}$ by using the backward difference scheme, in order to ensure that all the coefficients of $y_{j}^{(n)}$ are positive for $-M \leq j \leq M$. Then the descretization of the space-time fade in this case reads

$$
\begin{aligned}
& \tau^{-\beta} \sum_{m=0}^{n+1}(-1)^{m}\left(\begin{array}{l}
\beta \\
m
\end{array}\right)\left(y_{j}^{(n+1-m)}-y_{j}^{(0)}\right) \\
= & -\frac{a h^{-\alpha}}{2 \cos \frac{\alpha \pi}{2}} \sum_{k=0}(-1)^{k}\left(\begin{array}{l}
\alpha \\
k
\end{array}\right)\left(y_{j+1-k}^{(n)}+y_{j-1+k}^{(n)}\right) \\
& -\frac{b}{h}\left(y_{j}^{(n)}-y_{j-1}^{(n)}\right) .
\end{aligned}
$$


After using the scaling relation (5.10), I am going to separate the coefficients related to $k=0,1,2$ from the last summation and rearrange Equation (5.16) as

$$
\begin{aligned}
y_{j}^{(n+1)}= & b_{n} y_{j}^{(0)}+\sum_{m=2}^{n} c_{m} y_{j}^{(n+1-m)} \\
& +y_{j}^{(n)}\left(\beta-\frac{b \tau^{\beta}}{h}+\frac{a \mu}{\cos \frac{\alpha \pi}{2}}\left(\begin{array}{l}
\alpha \\
1
\end{array}\right)\right) \\
& +y_{j-1}^{(n)}\left(\frac{b \tau^{\beta}}{h}-\frac{a \mu}{2 \cos \frac{\alpha \pi}{2}}\left(1+\left(\begin{array}{l}
\alpha \\
2
\end{array}\right)\right)\right) \\
& -y_{j+1}^{(n)}\left(\frac{a \mu}{2 \cos \frac{\alpha \pi}{2}}\left(1+\left(\begin{array}{l}
\alpha \\
2
\end{array}\right)\right)\right) \\
& -\frac{a \mu}{2 \cos \frac{\alpha \pi}{2} \sum_{k \geq 3}(-1)^{k}\left(\begin{array}{l}
\alpha \\
k
\end{array}\right)\left(y_{j+1-k}^{(n)}+y_{j-1+k}^{(n)}\right)}
\end{aligned}
$$

To ensure that all the coefficients of $y_{j}^{(n)}$ are positive, the scaling relation must satisfy

$$
0<\mu \leq\left(\frac{-\beta+\frac{b \tau^{\beta}}{h}}{a \alpha}\right) \cos \frac{\alpha \pi}{2} .
$$

It is known as $1<\alpha<2$, the value of $\cos \frac{\alpha \pi}{2}<0$, and that ensure that $\mu \geq 0$. I can use the same symbols of the last subsection to write this equation in the form of the random walk as

$$
\begin{aligned}
y_{j}^{(n+1)}= & b_{n} y_{j}^{(0)}+\sum_{m=2}^{n} c_{m} y_{j}^{(n+1-m)}+p_{j, j} y_{j}^{(n)} \\
& +p_{j, j-1} y_{j-1}^{(n)}+p_{j, j+1} y_{j+1}^{(n)} \\
& +\sum_{k \geq 3}^{N}\left(p_{j, j-k} y_{j-k}^{(n)}+p_{j, j+k} y_{j+k}^{(n)}\right) .
\end{aligned}
$$

Again the summation of all the transition probabilities of this equation over all $j \in \mathbb{Z}$ is one. As in the previous, Equation (5.17) can be written in the same matrix form (5.13) where the diagonal elements of the matrix $A$ are defined as (see (5.19) below)

In the numerical calculations, I plot the path of the particle for different values of $t$. I choose the values of $\mu$ according to condition of each case. The situation as $\beta=1$ is the same as the previous cases. Now, I am going to prove the convergence of the discrete solution (5.18) in the Fourier-Laplace domain to the solution of the space-time fade as $1<\alpha \leq 2$. To do so, one has to shift the indices of $y_{j \pm 1 \mp k}$ and rewrite Equation (5.18) as

$$
\begin{aligned}
\sum_{m=0}^{n+1}(-1)^{m}\left(\begin{array}{c}
\beta \\
m
\end{array}\right)\left(y_{j}^{(n+1-m)}-y_{j}^{(0)}\right) & \left(-\frac{b \tau^{\beta}}{h}+\frac{a \alpha \mu}{\cos \frac{\alpha \pi}{2}}\right) y_{j}^{(n)}+\left(\frac{b \tau^{\beta}}{h}-\frac{a \mu}{2 \cos \frac{\alpha \pi}{2}}\right) y_{j-1}^{(n)} \\
& -\frac{a \mu}{2 \cos \frac{\alpha \pi}{2}} y_{j+1}^{(n)}-\frac{a \mu}{2 \cos \frac{\alpha \pi}{2}} \sum_{k \geq 2}(-1)^{k}\left(\begin{array}{c}
\alpha \\
|k+1|
\end{array}\right) y_{j-k}^{(n)}
\end{aligned}
$$

Again multiply each side by $z^{j}$ and sum over all $j$ and use all the identities of the last section, to get

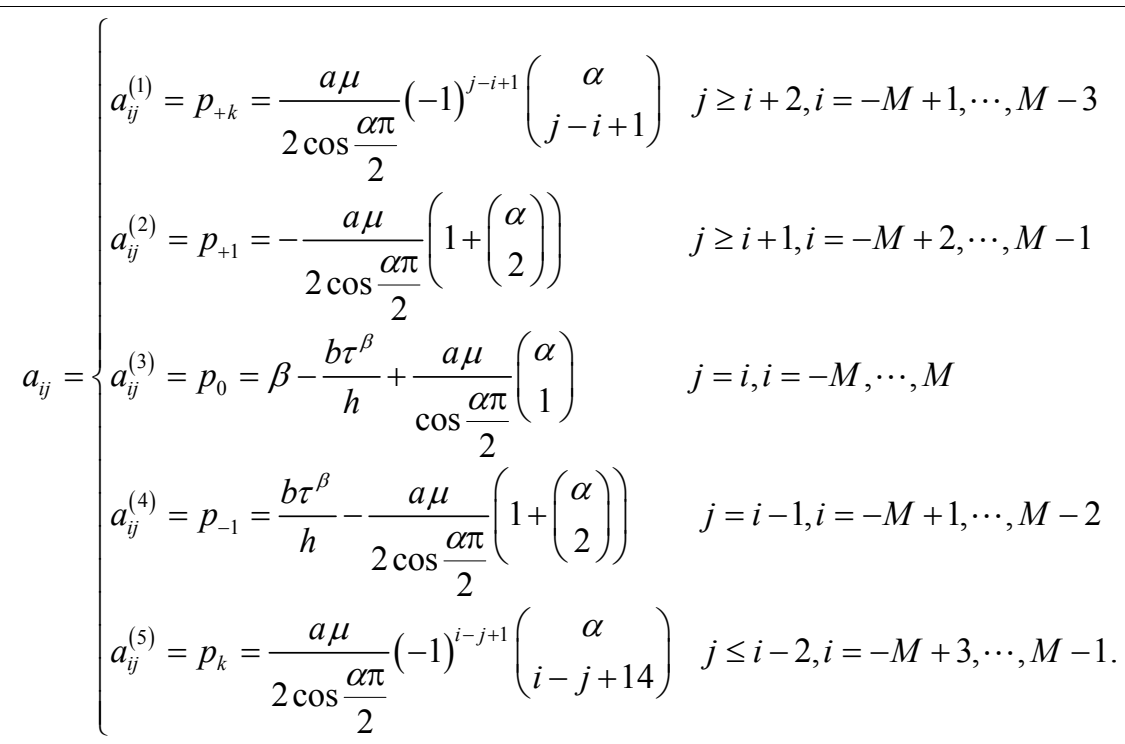




$$
\begin{aligned}
& \sum_{m=0}^{n+1}(-1)^{m}\left(\begin{array}{c}
\beta \\
m
\end{array}\right)\left(q_{n+1-m}(z)-q_{n}(0)\right) \\
= & \left(-\frac{b \tau^{\beta}}{h}+\frac{a \alpha \mu}{\cos \frac{\alpha \pi}{2}}\right) q_{n}^{(z)} \\
& +z q_{n}(z)\left(\frac{b \tau^{\beta}}{h}-\frac{a \mu}{2 \cos \frac{\alpha \pi}{2}}\right) \\
& -\frac{1}{z} q_{n}(z) \frac{a \mu}{2 \cos \frac{\alpha \pi}{2}} \\
& -\frac{a \mu}{2 \cos \frac{\alpha \pi}{2}} q_{n}(z)\left(\frac{1}{z}(1-z)^{\alpha}+z\left(1-\frac{1}{z}\right)^{\alpha}\right) .
\end{aligned}
$$

Replace $z=\mathrm{e}^{i \kappa h}$, where, it is clear that

$$
\begin{aligned}
& \frac{\mu}{2 \cos \frac{\alpha \pi}{2}}\left(\mathrm{e}^{-i \kappa h}\left(1-\mathrm{e}^{i \kappa h}\right)^{\alpha}+\mathrm{e}^{i \kappa h}\left(1-\mathrm{e}^{-i \kappa h}\right)^{\alpha}\right) \\
= & \frac{(\kappa h)^{\alpha}}{\cos \frac{\alpha \pi}{2}} \cos \left(\frac{\alpha \pi}{2}-\kappa h\right) \approx \kappa^{\alpha} \tau^{\alpha} \quad \text { as } h \rightarrow 0
\end{aligned}
$$

So one gets

$$
\begin{aligned}
& \sum_{m=0}^{n+1}(-1)^{m}\left(\begin{array}{c}
\beta \\
m
\end{array}\right)\left(q_{n+1-m}\left(\mathrm{e}^{i \kappa h}\right)-q_{n}(0)\right) \\
= & \left(i b \kappa \tau^{\beta}-a \kappa^{\alpha} \tau^{\beta}\right) q_{n}\left(\mathrm{e}^{i \kappa h}\right),
\end{aligned}
$$

Now, multiply both sides by $\zeta^{n},|\zeta| \leq 1$, and sum over all $n$, to get

$$
\begin{aligned}
& \frac{(1-\zeta)^{\beta}}{\zeta}\left(Q(z, \zeta)-\frac{1}{1-\zeta}\right) \\
= & \zeta\left(i b \kappa \tau^{\beta}-a \kappa^{\alpha} \tau^{\beta}\right) Q\left(\mathrm{e}^{i \kappa h, \zeta}\right)
\end{aligned}
$$

Finally, replace $\zeta$ by $\mathrm{e}^{-s \tau}$, solve for $Q\left(\mathrm{e}^{i \kappa h}, \mathrm{e}^{-s \tau}\right)$, and multiply both sides by $\tau$, you will get the desired aim $\tau Q\left(\mathrm{e}^{i \kappa h}, \mathrm{e}^{-s \tau}\right)=\hat{\tilde{u}}(\kappa, s)$, Equation (5.2). Then the discrete solution converges to the solution of its corresponding space-time fade in the Fourier-Laplace domain.

\section{The Discretization of the Space-Time Fade as $\alpha=1,0<\beta \leq 1$}

The Cauchy fractional ade needs a special treatment. I am going to prove first the convergence of the discrete solution. Therefore, the Fourier-Laplace transformation of the space-time fade (2.1), as $\alpha=1$, reads

$$
\hat{\tilde{u}}(\kappa, s)=\frac{s^{\beta-1}}{s^{\beta}+a|\kappa|-i b \kappa} .
$$

This case is related to the Cauchy distribution and one cannot use the Grünwald-Letnikov discretization of $D_{0}^{1}$ at Equations (5.7) and (5.8) because the denominator is zero and $c_{ \pm} \rightarrow 0$ for $\alpha=1$. Instead of GrünwaldLetnikov discretization, one must use the descretization used in [33]. The authors of [33] deduced the descretization of $D_{0}^{1}$ from the Cauchy density $p_{1}(x, 0)=\frac{1}{\pi} \frac{1}{1+x^{2}}$, see [6] for more information. They replaced the factor $(-1)^{k}\left(\begin{array}{l}\alpha \\ k\end{array}\right), k \in \mathbb{Z}$, in Equations (5.7) and (5.8) by $\frac{-2}{\pi h}$ for $k=0$, and $\frac{1}{\pi h|k|(|k|+1)}$ for $k \neq 1, k \in \mathbb{Z}$. Therefore by using the scaling parameter

$$
\mu=\frac{\tau^{\beta}}{h},
$$

one gets

$$
\begin{aligned}
& \sum_{m=0}^{n+1}(-1)^{m}\left(\begin{array}{c}
\beta \\
m
\end{array}\right)\left(y_{j}^{(n+1-m)}-y_{j}^{(0)}\right) \\
= & \frac{-2 a \mu}{\pi} y_{j}^{(n)}+\frac{a \mu}{\pi} \sum_{k \geq 1} \frac{1}{k(k+1)}\left(y_{j+k}^{(n)}+y_{j-k}^{(n)}\right) \\
& -\frac{b \mu}{2}\left(y_{j+1}^{(n)}-y_{j-1}^{(n)}\right) .
\end{aligned}
$$

As the previous cases, $y_{j}^{(n+1)}$ can be written in the form of a random walk with a memory as

$$
\begin{aligned}
y_{j}^{(n+1)}= & b_{n} y_{j}^{(0)}+\sum_{m=2}^{n} c_{m} y_{j}^{(n+1-m)}+\left(\beta-\frac{2 a \mu}{\pi}\right) y_{j}^{(n)} \\
& +\left(\frac{a \mu}{2 \pi}-\frac{b \mu}{2}\right) y_{j+1}^{(n)} \\
& +\left(\frac{a \mu}{2 \pi}+\frac{b \mu}{2}\right) y_{j-1}^{(n)} \\
& +\frac{a \mu}{\pi} \sum_{k \geq 2} \frac{1}{k(k+1)}\left(y_{j+k}^{(n)}+y_{j-k}^{(n)}\right) .
\end{aligned}
$$

To have all the coefficients of $y_{j}^{(n)}$ are positive, one should restrict the values $\mu$ as $\mu \leq \frac{\pi \beta}{2 a}$. Following [33], it can be proved that the summations of the transition probabilities of Equation (6.4) are summed to one and one can easily write it in the form of a random walk as the previous cases. The last equation could also be written on the same matrix form (5.13). Where $A$ is a fifth diagonal matrix whose diagonal elements are defined as 


$$
a_{i j}=\left\{\begin{array}{lll}
a_{i j}^{(1)} & =\frac{a \mu}{\pi} \frac{1}{(j-i)(j-i+1)} & j \geq i+2, i=-M+1, \cdots, M-3 \\
a_{i j}^{(2)}=\frac{a \mu}{2 \pi}-\frac{b \mu}{2} & j \geq i+1, i=-M+2, \cdots, M-1 \\
a_{i j}^{(3)}=\beta-\frac{2 a \mu}{\pi} & j=i, i=-M,, \cdots, M-1 \\
a_{i j}^{(4)}=\frac{a \mu}{2 \pi}+\frac{b \mu}{2} & j=i-1, i=-M+1, \cdots, M-2 \\
a_{i j}^{(5)}=\frac{a \mu}{\pi} \frac{1}{(i-j)(i-j+1)} & j \leq i-2, i=-M+3, \cdots, M-1 .
\end{array}\right.
$$

The numerical result of this model is discussed in the next section. Noting that the coefficient $a$ and $b$ play here a significant role. To prove the convergence, multiply each side of Equation (6.3) by $z^{j}$ and sum over all $j$, then use the identity

$$
\sum_{j=-\infty}^{\infty} \frac{1}{j(j+1)} z^{j}=1-\left(1-\frac{1}{z}\right) \log (1-z),
$$

to get

$$
\begin{aligned}
& \sum_{m=0}^{n+1}(-1)^{m}\left(\begin{array}{l}
\beta \\
m
\end{array}\right)\left(q_{n+1-m}(z)-1\right) \\
= & -\frac{a \mu q_{n}(z)}{\pi}\left(\left(1-\frac{1}{z}\right) \log (1-z)+(1-z) \log \left(1-\frac{1}{z}\right)\right)(6.6) \\
& -\frac{b \mu q_{n}(z)}{2}\left(\frac{1}{z}-z\right) .
\end{aligned}
$$

Replace, $z$ by $\mathrm{e}^{i \kappa h}$, and use the identity

$\left(1-\mathrm{e}^{-i \kappa h}\right) \log \left(1-\mathrm{e}^{i \kappa h}\right)+\left(1-\mathrm{e}^{i \kappa h}\right) \log \left(1-\mathrm{e}^{-i \kappa h}\right)$

$=2\left\{(1-\cos \kappa h) \log \left|1-\mathrm{e}^{i \kappa h}\right|+\sin (\kappa h) \arctan \frac{\sin \kappa h}{1-\cos \kappa h}\right\}$

$\approx \pi \kappa h \quad$ as $h \rightarrow 0$.

Finally replace, $\zeta$ by $\mathrm{e}^{-s \tau}$ and take the limit as $\tau \rightarrow 0$, you get $\tau Q\left(\mathrm{e}^{i \kappa h}, \mathrm{e}^{-s \tau}\right)=\hat{\tilde{u}}(\kappa, s)=$, Equation (6.1).

\section{Numerical Results}

In this section, I give the numerical approximate solutions for Equation (2.1). I give the evolution of $y^{(n)}=y\left(t_{n}\right)$ with different values of $t_{n}$ such, different values of the space fractional order $\alpha$ and different values of the time fractional order $\beta$. I fix the values of $M=20$ while $N=2 M+1$ and $h=2 / N$, with the initial condition $y^{(0)}=\{0, \cdots, 1, \cdots, 0\}$ as it must satisfy $\sum_{j=0}^{N} y_{j}^{(0)}=1$. Since $\left\{t_{0}, t_{1}, t_{2}, \cdots\right\}=\{0,1,2, \cdots\}$, then the iteration index $n=\frac{t_{n}}{\tau}$ while $\tau$ is calculated from the scaling parameter of the specified model and its values are varying according to the restriction put on $\mu$. Since I used the explicit discrete scheme, therefore, sometimes I need a huge number of steps for calculating $y^{(n)}=y\left(t_{n}\right)$ specially for $\beta \leq 1$. I calculate most of the numerical results for $a=1$ and $b=1$ but as $0<\alpha<1$ and $1<\alpha$ $<2$ I used $b<1$ to ensure that all the elements must be $a_{i j} \geq 0$, because $A$ is probability transition matrices. To ease comparing the numerical results, I wrote the values of $\alpha, \beta$ and their corresponding $\mu$ with the values of $t$ at the figure. The classical case, i.e. as $\alpha=2$ and $\beta=1$, is plotted at Figures 1 and 2 and one can observe how rapidly the paths diffuse as the time increases. The time-fractional ade is simulated at Figures 3 and 4, i.e. for $\alpha=2$ and $\beta<1$. Figures 5, 6 are corresponding to the space-time fractional ade as $0<$ $\alpha<1$ and $\beta=1$. While Figures 7 and 8 are denoted for $0<\alpha<1$ and $\beta<1$. Figures 9 and $\mathbf{1 0}$ are corresponding to $1<\alpha<2$ and $\beta=1$ at Figures 11 and 12 for $1<$ $\alpha<2$ and $\beta<1$. Finally, I plot the space-time fractional ade as $\alpha=1$ and $\beta \leq 11$ is plotted at Figures 13 and 14. The numerical results of this paper as $1<\alpha \leq 2$ and $\beta=1$ are consistent with the results at [17]. The numerical solution for the case $\alpha=2$ and $\beta=1$, i.e., the classical case in this paper is seemed perfect when comparing with the other references as it has been

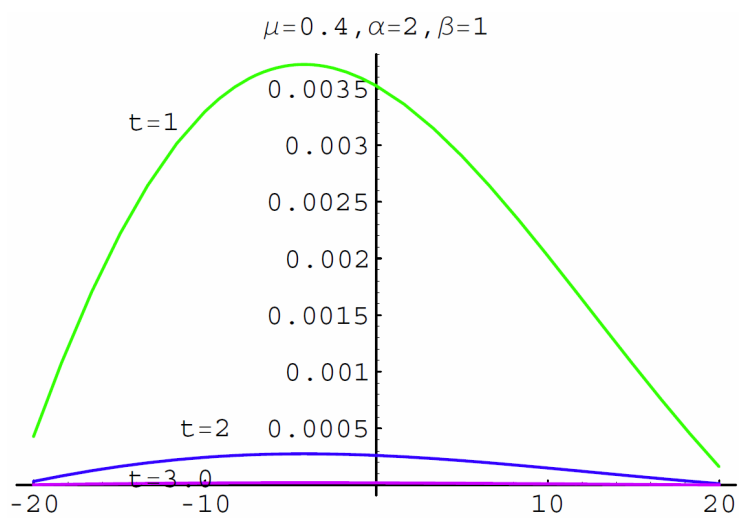

Figure 1. The classical case. 


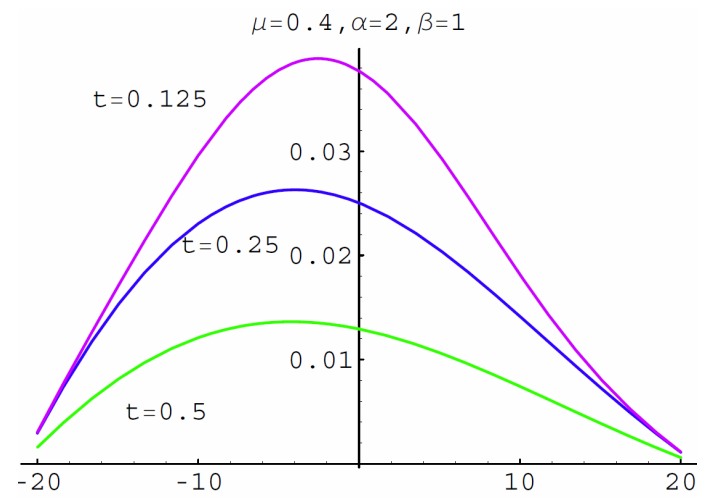

Figure 2. Classical case.

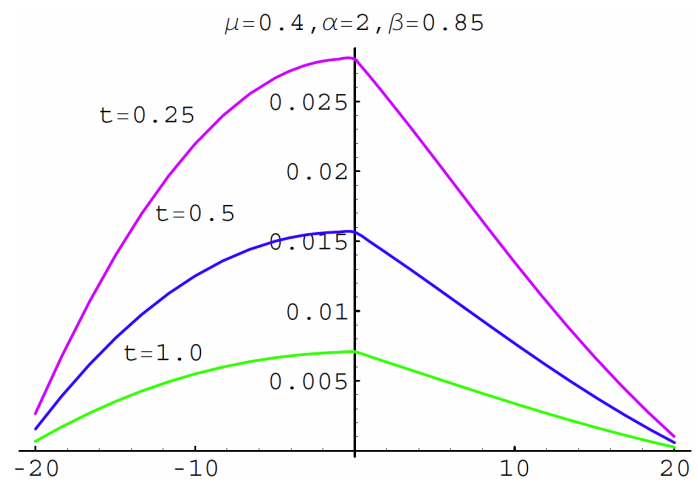

Figure 3. Time-fractional.

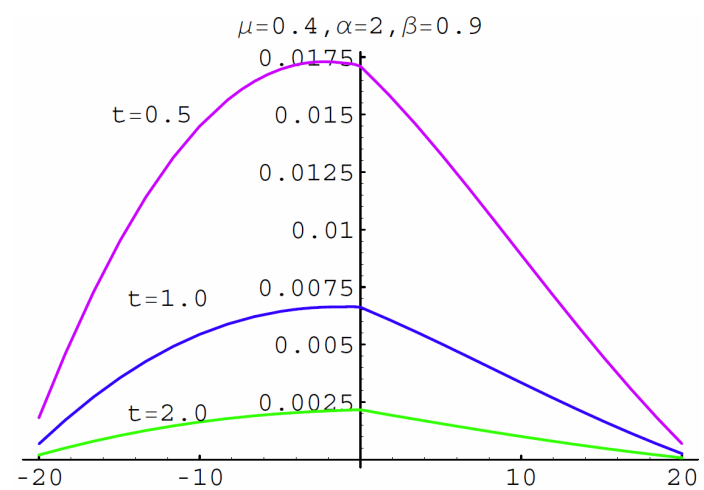

Figure 4. Space-time-fractional.

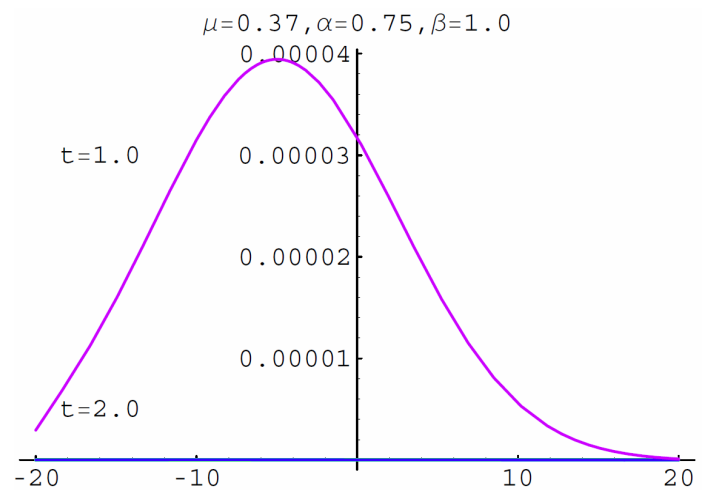

Figure 5. Space-fractional.

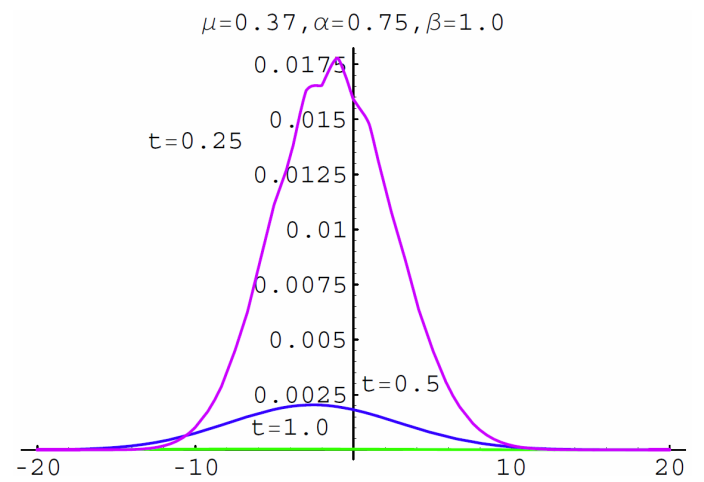

Figure 6. Space-fractional.

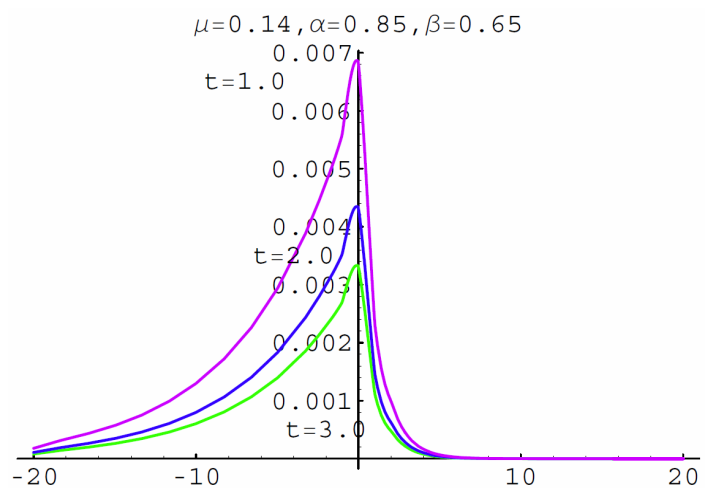

Figure 7. Space-time-fractional.

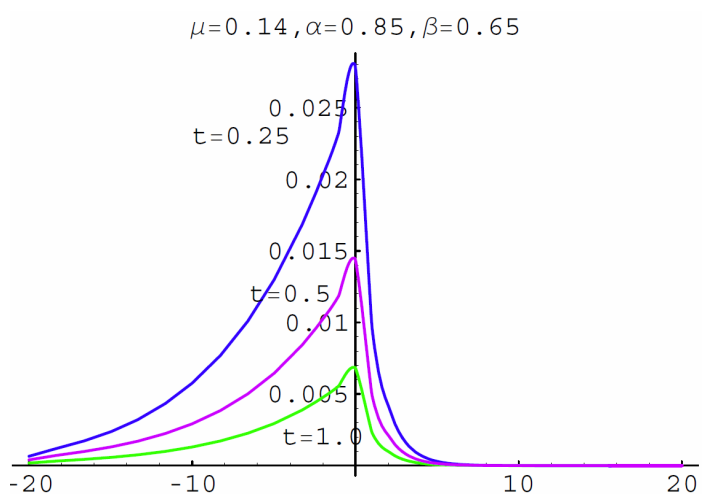

Figure 8. Space-time fractional.

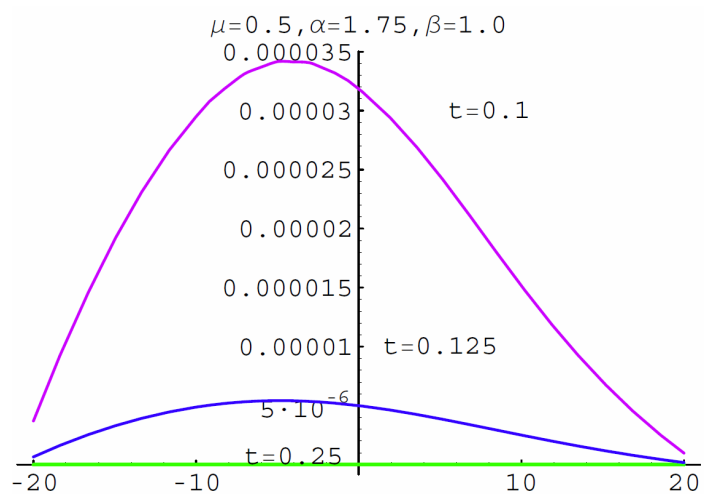

Figure 9. Space-fractional. 


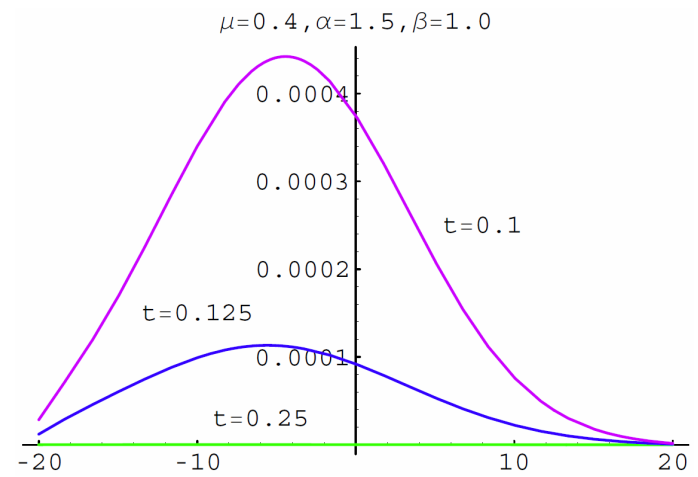

Figure 10. Space-fractional.

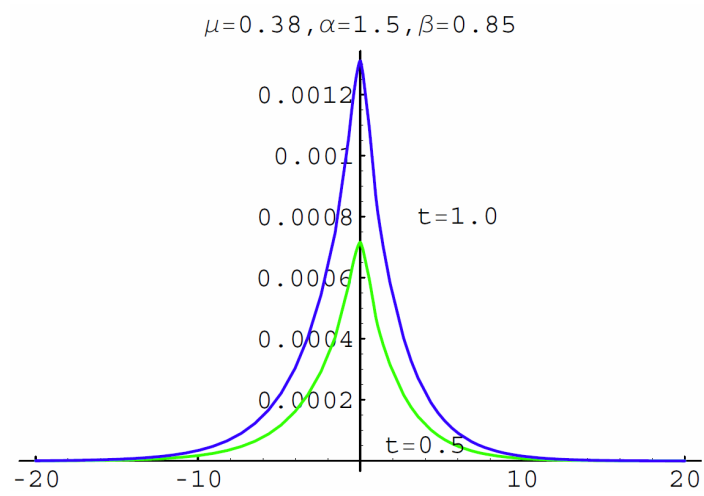

Figure 11. Space-time fractional.

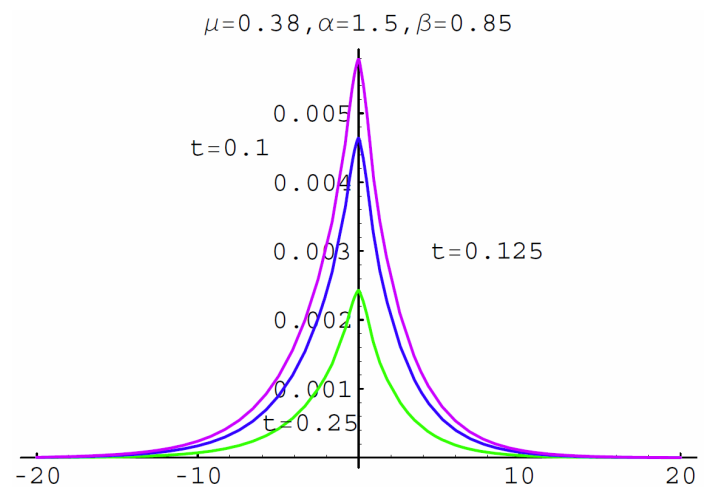

Figure 12. Space-time fractional.

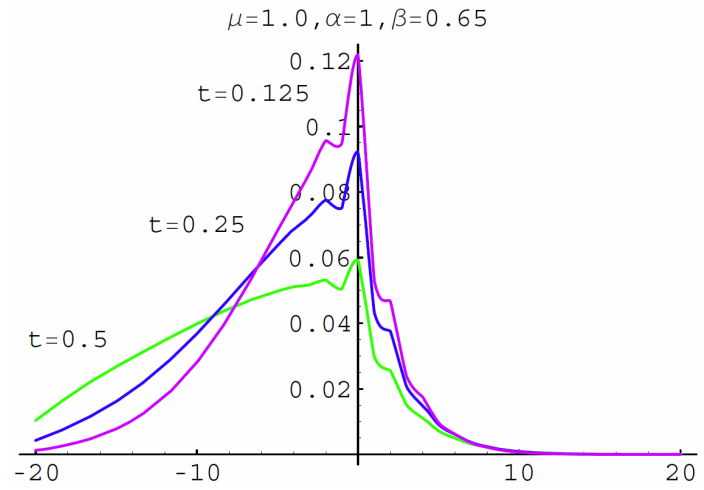

Figure 13. The singular case.

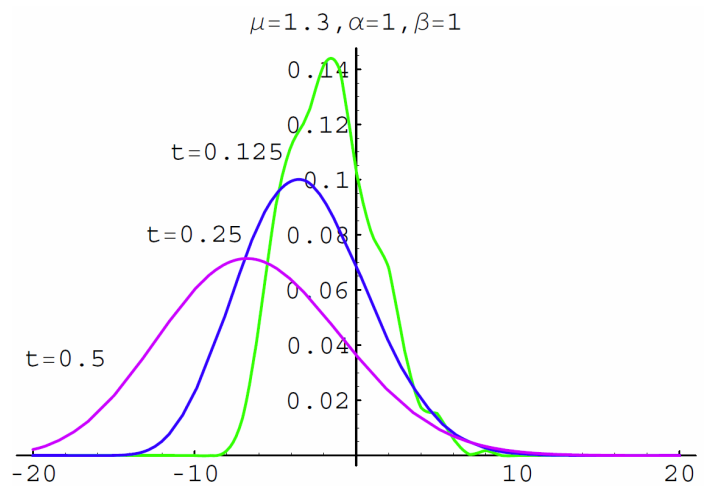

Figure 14. The singular case.

discussed by many authors. The pates as $\beta=1$ are different than the paths corresponding to $\beta<1$. The pathes for $\beta<1$ need a huge number of time steps to calculate them as I use the explicit difference methods.

\section{REFERENCES}

[1] K. S. Miller and B. Ross, "An Introduction to the Fractional Calculus and Fractional Differential Equations," John Wily and Sons, INC., New York, Chichester, Brisbane, Toronto, Singapore, 1993.

[2] I. Podlubny, "Fractional Differential Equations," Academic Press, San Diego, Boston, New York, London, Sydney, Tokyo, Toronto, 1999.

[3] S. G. Samko, A. A. Kilbas and O. I. Marichev, "Fractional Integrals and Derivatives (Theory and Applications)," Gordon and Breach, New York, London, and Paris, 1993.

[4] R. Gorenflo and F. Mainardi, "Fractional Calculus: Integral and Differential Equations of Fractional Order," In: A. Carpinteri and F. Mainardi, Eds., Fractals and Fractional Calculus in Continuum Mechanics, Springer Verlag, Wien and New York, 1997, pp. 223-276. http://www.fracalmo.org

[5] N. U. Prabhu, "Stochastic Processes (Basic Theory and Its Applications)," The Macmillan Company, New York, Collier-Macmillan Limited, London, 1965.

[6] E. A. Abdel-Rehim, "Modelling and Simulating of Classical and Non-Classical Diffusion Processes by Random Walks," Mensch\&Buch Verlag, 2004. http://www.diss.fu-berlin.de/2004/168/index.html

[7] W. Feller, 'On a Generalization of Marcel Riesz' Potentials and the Semi-Groups Generated by Them," In: Meddelanden Lunds Universitetes Matematiska Seminarium (Comm. Sém. Mathém. Université de Lund), Tome Suppl. dédié a M. Riesz, Lund, 1952, pp. 73-81.

[8] M. M. Meerschaert and C. Tadjeran, "Finite Difference Approximations for Fractional Advection-Dispersion Flow Equation," Journal of Computational and Applied Mathematics, Vol. 172, No. 1, 2004, pp. 65-77. http://dx.doi.org/10.1016/j.cam.2004.01.033

[9] D. A.Benson, S. W. Wheatcraft and M. M. Meerschaert, "Application of a Fractional Advection-Dispersion Equation," Water Resource Research, Vol. 36, No. 6, 2000, pp. 
1403-1412. http://dx.doi.org/10.1029/2000WR900031

[10] D. A. Benson, R. Schumer, M. M. Meerschaert and S. W. Wheatcraft, "Fractional Dispersion, Lévy Motion, and the MADE Tracer Tests," Transport in Porous Media, Vol. 42, No. 1-2, 2001, pp. 211-240. http://dx.doi.org/10.1023/A:1006733002131

[11] B. Baeumer, D. A. Benson, M. M. Meerschaert and S. W. Wheatcraft, "Subordinated Advection-Dispersion Equation for Contaminant Transport," Water Resource Research, Vol. 37, No. 6, 2001, pp. 1543-1550.

[12] R. Schumer, M. M. Meerschaert and B. Baeumer, "Fractional Advection-Dispersion Equations for Modeling Transport at the Earth Surface," Journal of Geophysical research, Vol. 114, No. F4, 2009. http://dx.doi.org/10.1029/2008JF001246

[13] F. Huang and F. Liu, "The Fundamental Solution of the spaace-time Fractional advection-dispersion Equation," Journal of Applied Mathematics and Computing Vol. 18, No. 1-2, 2005, pp. 339-350.

[14] Y.-S. Park and J.-J. Baik, "Analytical Solution of the Advection-Diffusion Equation for a Ground-Level Finite Area Source," Atomspheric Environment, Vol. 42, No. 40, 2008, pp. 9603-9069. http://dx.doi.org/10.1016/j.atmosenv.2008.09.019

[15] D. K. Jaiswal, A. Kumar and R. R. Yadav, "Analytical Solution to the One-Dimensional Advection-Diffusion Equation with Temporally Dependent Coefficients," Journal of Water Resource and Protection, Vol. 3, No. 1, 2011, pp. 76-84. http://dx.doi.org/10.4236/jwarp.2011.31009

[16] Y. Xia, J. C. Wu and L. Y. Zhou, "Numerical Solutions of Time-Space Fractional Advection-Dispersion Equations," ICCES, Vol. 9, No. 2, 2009, pp. 117-126.

[17] Q. Liu, F. Liu, I. Turner and V. Anh, "Approximation of the Lévy-Feller Advection-Dispersion Process by Random Walk and Finite Difference Method," Journal of Computational Physics, Vol. 222, No. 1, 2007, pp. 57-70. http://dx.doi.org/10.1016/j.jcp.2006.06.005

[18] F. Mainardi, Y. Luchko and G. Pagnini, "The Fundamental Solution of the Space-Time Fractional Diffusion Equation," Fractional Calculus and Applied Analysis, Vol. 4, No. 2, 2001, pp. 153-192. www.fracalmo.org

[19] R. Gorenflo, F. Mainardi, D. Moretti and P. Paradisi, "Time-Fractional Diffusion: A Discrete Random Walk Approach," Nonlinear Dynamics, Vol. 29, No. 1-4, 2002, pp. 129-143. http://dx.doi.org/10.1023/A:1016547232119

[20] R. Gorenflo and E. A. Abdel-Rehim, "Discrete Models of Time-Fractional Diffusion in a Potential Well," Fractional Calculus and Applied Analysis, Vol. 8, No. 2, 2005, pp. 173-200.
[21] R. Gorenflo and E. A. Abdel-Rehim, "From Power Laws to Fractional Diffusion: The Direct Way," Vietnam Journal of Mathematics, Vol. 32, No. SI, 2004, pp. 65-75.

[22] R. Gorenflo and E. A. Abdel-Rehim, "Convergence of the Grünwald-Letnikov Scheme for Time-Fractional Diffusion," Journal of Computational and Applied Mathematics, Vol. 205, No. 2, 2007, pp. 871-881. http://dx.doi.org/10.1016/i.cam.2005.12.043

[23] R. Gorenflo and E. A. Abdel-Rehim, "Simulation of Continuous Time Random Walk of the Space-Fractional Diffusion Equations," Journal of Computational and Applied Mathematics, Vol. 222, No. 2, 2008, pp. 274-285. http://dx.doi.org/10.1016/j.cam.2007.10.052

[24] E. A. Abdel-Rehim, "From the Ehrenfest Model to TimeFractional Stochastic Processes," Journal of Computational and Applied Mathematics, Vol. 233, No. 2, 2009, pp. 197-207. http://dx.doi.org/10.1016/j.cam.2009.07.010

[25] A. I. Saichev and G. M. Zaslavsky, "Fractional Kinetic Equations: Solutions and Applications," Chaos, Vol. 7, No. 4, 1997, pp. 753-764. http://dx.doi.org/10.1063/1.166272

[26] J. A. Goldstein, "Semigroups of Linear Operators and Applications," Oxford University Press, Oxford and New York, 1985.

[27] N. Jacob, "Pseudo-Differential Operators and Markov Processes," Akademie Verlag, Berlin, 1996.

[28] R. Metzler, J. Klafter and I. M. Sokolov, "Anomalous Transported in External Fields: Continuous Time Random Walks and Fractional Diffusion Equations Extended,' Physical Review E, Vol. 48, No. 2, 1998, pp. 1621-1633. http://dx.doi.org/10.1103/PhysRevE.58.1621

[29] W. Feller, "An Introduction to Probability Theory and Its Applications," Vol. 2, Johon Wiley and Sons, New York, London, Sydney, Toronto, 1971.

[30] M. Kac, "Random Walk and the Theory of Brownian Motion," The American Mathematical Monthly, Vol. 54, No. 7, 1947, pp. 369-391. http://dx.doi.org/10.2307/2304386

[31] K. B. Oldham and J. Spanier, "The Fractional Calculus," Vol. 3 of Mathematics in Science and Engineering, Academic Press, New York, 1974.

[32] R. Gorenflo and F. Mainardi, "Random Walk Models for Space-Fractional Diffusion Processes," Fractional Calculus and Applied Analysis, Vol. 1, No. 2, 1998, pp. 167190.

[33] R. Gorenflo and F. Mainardi, "Approximation of LévyFeller Diffusion by Random Walk," Journal of Analysis and its Applications (ZAA) Vol. 18, No. 2, 1999, pp. 231246. 